\title{
Imaging of Radiolucent Jaw Lesions
}

\author{
Abdellatif Bali, MD ${ }^{1} \quad$ Filip M. Vanhoenacker, MD, PhD $D^{1,2,3}$ Charlotte Vanhoenacker, MD ${ }^{4}$ \\ Anja Bernaerts, MD ${ }^{5}$
}

${ }^{1}$ Department of Radiology, Antwerp University Hospital, Edegem, Belgium

Address for correspondence Abdellatif Bali, MD, Department of

${ }^{2}$ Department of Radiology, AZ Sint-Maarten, Mechelen, Belgium Radiology, University Hospital of Antwerp UZA, Wilrijkstraat 10, 2650

3 Department of Radiology, Ghent University Hospital, Ghent, Belgium

${ }^{4}$ Department of Radiology, University Hospitals Leuven, Leuven, Belgium

${ }^{5}$ Department of Radiology, GZA Sint-Augustinus, Antwerp, Belgium Edegem, Belgium (e-mail: abdellatif.bali@gmail.com).

Semin Musculoskelet Radiol 2020;24:549-557.

\begin{abstract}
Keywords

- jaws

- tumor and tumor-like lesions

- radiolucency

- cone beam computed tomography

- imaging

Radiolucent lesions in the jaw bones comprise a whole spectrum of odontogenic and nonodontogenic lesions. Although the imaging appearance is not always specific, careful radiologic analysis may contribute to characterization of these lesions. A useful approach is to first analyze the absence or presence of a relationship of the lesion to the teeth. The relation may be either near the tooth apex or crown of the tooth. Other lesions may or may not show any specific anatomical location. After analysis of the primary location of the lesion, additional criteria that may help in further imaging characterization are lesion demarcation and morphology, involvement of the cortex and periosteum, and soft tissue changes. This article describes the most characteristic and prevalent radiolucent lesions of the jaws at each location. In routine clinical practice, cone beam computed tomography is sufficient for appropriate lesion characterization, although magnetic resonance imaging may be useful in selected cases.
\end{abstract}

\section{Lesions with Preferential Periapical Location}

\section{Radicular Cyst}

A radicular cyst, a cyst with an epithelial lining, is also known as a periodontal, periapical, or dental cyst. It is the most common odontogenic cyst. A radicular cyst arises from the periodontal ligament usually secondary to low-grade inflammation and infection. It therefore occurs most commonly at the apex of a nonvital tooth. In addition to caries, it can also result from trauma. These two mechanisms may lead to necrosis of the pulp cavity and may subsequently spread to the tooth apex, with development of a periapical granuloma or periapical abscess (-Fig. S1). This leads secondary to the formation of a radicular cyst. ${ }^{1}$

A radicular cyst can occur at any age but is most common in the third to fifth decade, without any sex predominance. ${ }^{2}$ It is most frequently (60\%) found in the maxilla and preferentially at the incisors and canines. Panoramic views or cone beam computed tomography (CBCT) shows a radiolucent, unilocular lesion, with well-defined sclerotic margins (-Fig. 1 and -Fig. S2). If secondarily infected, the lesion has ill-defined borders on imaging and typically is accompanied by pain. ${ }^{3} \mathrm{~A}$ radicular cyst may invaginate into the maxillary sinus. Resorption or displacement of adjacent teeth occasionally can occur. ${ }^{4}$ A radicular cyst is typically homogeneously isointense on T1-weighted images and homogeneously hyperintense on T2-weighted images, and it does not show contrast enhancement. ${ }^{4}$ Cortical breakthrough is typically absent, but cortical bone remodeling may occur secondary to the slowly expanding cyst. No surrounding periosteal reaction or soft tissue changes is observed. $^{3}$

\section{Odontogenic Keratocyst}

An odontogenic keratocyst (OKC), formerly known as a keratocytic odontogenic tumor, is the most aggressive of all jaw cysts and represents 3 to $11 \%$ of cystic lesions of the jaw., ${ }^{5,6}$ The two subtypes are "primordial-origin OKC" and "dentigerousorigin OKC" that account for $60 \%$ and $40 \%$, respectively. ${ }^{5}$ Primordial-origin OKC arises from dental lamina rests, an
Issue Theme Facial and Dental Musculoskeletal Imaging; Guest Editor, Filip M. Vanhoenacker, MD, PhD
Copyright (c) 2020 by Thieme Medical Publishers, Inc., 333 Seventh Avenue, New York, NY 10001, USA. Tel: +1(212) 760-0888.
DOI https://doi.org/ 10.1055/s-0040-1705118. ISSN 1089-7860. 


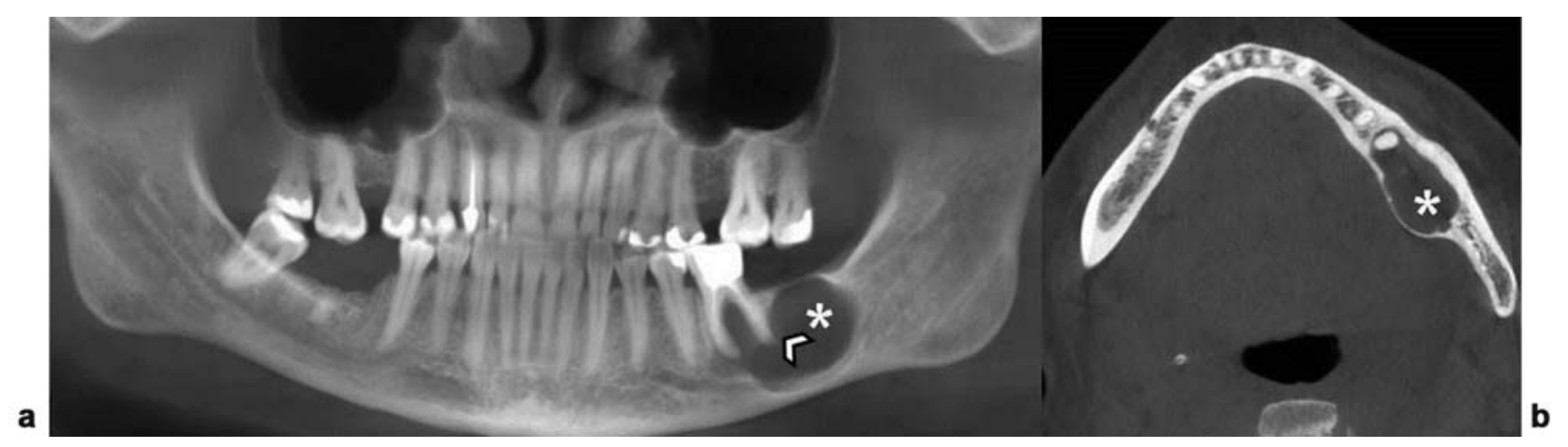

Fig. 1 Cone beam computed tomography of radicular cyst. (a) Panoramic reformatted image. (b) Axial reformatted image. Note a welldelineated radiolucent lesion (asterisk) with an intimate relationship with the apex of tooth 36 . There is limited resorption of the adjacent tooth apex (arrow).

epithelial structure found in the primitive oral mucosal epithelium responsible for tooth formation or from the basal cells of oral epithelium. Dentigerous-origin OKC, in contrast, arises from reduced enamel epithelium of the dental follicle. ${ }^{5}$

OKC is most common in the second to fourth decade. A slight male predominance was reported. ${ }^{4}$ In cases of multiple OKC, or when it occurs in children, the Gorlin-Goltz syndrome should be considered (also known as the basal cell nevus syndrome). ${ }^{7}$ Potential manifestations of this syndrome include multiple OKCs, skin basal cell carcinomas, various skeletal abnormalities, craniofacial anomalies, oropharyngeal anomalies, ophthalmic anomalies, and lamellar falx calcifications. ${ }^{7}$

The name OKC is derived from the presence of keratin within the cystic content. The mandible is affected twice as frequently as the maxilla. Most lesions (75\%) are located in the posterior mandible with half of them at the mandibular angle. ${ }^{8}$ OKCs may be located in a periapical position (33\%), in a pericoronal position (21\%), or in a lateral root position (19\%). In $\sim 27 \%$ of cases, there is not even any relationship with any dental structures. ${ }^{9}$

A systematic review of the literature by MacDonaldJankowski showed that patients of East Asian origin mostly present with swelling and pain, whereas discharge and numbness of the inferior alveolar nerve are more frequently seen in Latin Americans. ${ }^{10}$

On plain radiographs or $\mathrm{CBCT}$, the cyst margins are usually well delineated and may have a scalloped contour. They often remodel and/or displace the mandibular canal. They are more often unilocular than multilocular. ${ }^{4}$ Computed tomography (CT) and magnetic resonance imaging (MRI) (-Fig. 2) have an important role in differentiating OKC from ameloblastomas and other cystic lesions in the maxillomandibular region, which is important because of the high recurrence rate of OKC. Because of its paste-like consistency, ОКC may show high attenuation areas on CT.

Another feature that can be useful to differentiate OKC from other lesions is the tendency to grow within the medullary cavity in a predominant anteroposterior (mesiodistal) direction while causing minimal cortical expansion. ${ }^{11}$ On the contrary, a predominant buccal-lingual expansion is seen in ameloblastomas. Whereas on MRI, an ameloblastoma is hypointense on T1-weighted images and hyperintense on
T2-weighted images, OKC can have different signal intensity patterns. The keratin in OKC causes T1-shortening that leads to a hypointense to mildly hyperintense appearance relative to muscle on T1-weighted images. ${ }^{11}$ OKC typically do not enhance except for a thin peripheral rim, whereas a nodular enhancement pattern of the cyst wall rather suggests an ameloblastoma. ${ }^{5,12}$ Keratin also causes diffusion restriction with a high signal on b-value 1,000 diffusion-weighted images (DWIs) and a low signal on apparent diffusion coefficient (ADC) maps (- Fig. S3). ${ }^{1}$ Also the use of diffusion kurtosis imaging could be a helpful technique to differentiate these two entities. ${ }^{13}$

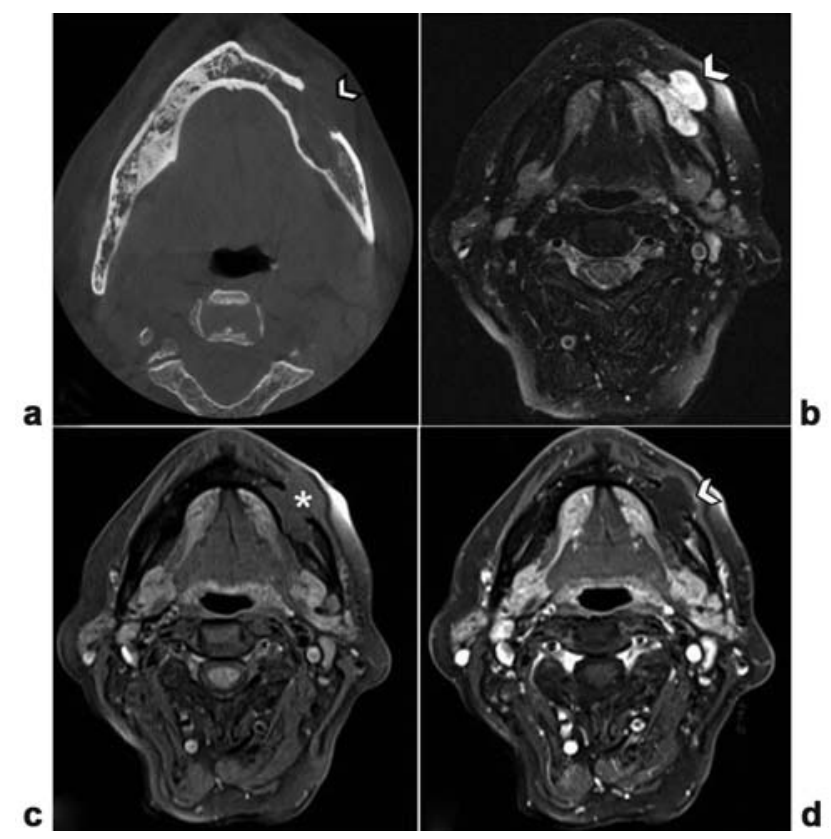

Fig. 2 Odontogenic keratocyst. (a) Axial computed tomography image in bone window shows an osteolytic lesion in the left body of the mandible causing cortical breakthrough of the vestibular cortex (white arrow). (b) Fat-suppressed T2-weighted image shows a T2-hyperintense lesion in the left mandible, with cortical breakthrough. This lesion has a characteristic mesiodistal expansion. The small intralesional hypointense foci correspond to keratin. Axial T1-weighted image with fat saturation (c) before and (d) after intravenous gadolinium administration show faint rim enhancement (white arrow) of the osteolytic lesion (asterisk). 


\section{Lateral Periodontal Cyst}

A lateral periodontal cyst (LPC) is defined as a nonkeratinized and noninflammatory developmental cyst, located adjacent to the root of a vital tooth. ${ }^{14}$ It affects young adults, men more than women. ${ }^{15}$ It is more common in the mandible than in the maxilla, usually at the level of the mandibular premolars, lateral to the tooth root (which explains the name).

On panoramic views, an LPC is typically a well-defined unilocular radiolucency. A multiloculated morphology is occasionally seen. ${ }^{4}$ Large cysts may displace adjacent teeth (-Fig. S4). If the lesion is small and with small cystic contents, MRI could be helpful for further differentiation with OKCs, small ameloblastoma, carcinoma, or neurofibroma. ${ }^{4}$

\section{Acute Osteomyelitis}

Acute osteomyelitis of the jaws is most often secondary to direct extension of an acute pulpal infection without the formation of a granuloma or from the acute exacerbation of a chronic periapical lesion. It may also occur following penetrating trauma or various surgical procedures, such as wisdom teeth extraction.

Plain radiographs are often unremarkable during the first 7 to 14 days after the onset of the disease except for a subtle widening of the periodontal space around the root apex or generalized osteoporosis. ${ }^{5}$ After 2 weeks, panoramic radiographs may show an ill-defined osteolytic lesion.

Contrast-enhanced CT ( - Fig. 3 and - Fig. 55) is useful for defining possible sinus tracts, periosteal reactions, or abscess formation. MRI is more sensitive than $\mathrm{CT}$, however, in detecting bone marrow and soft tissue involvement, and it

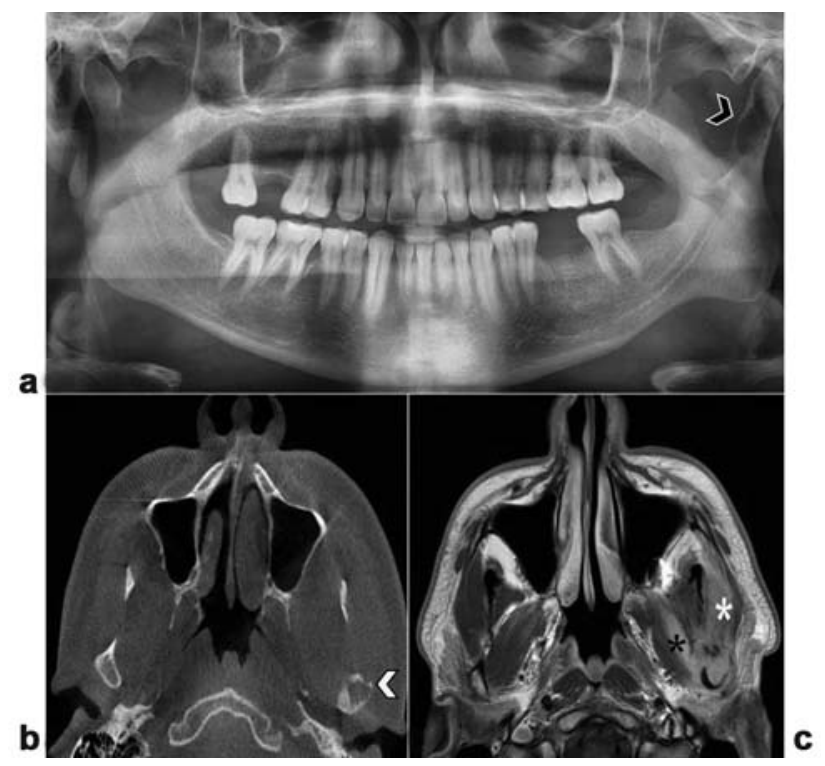

Fig. 3 Acute osteomyelitis. (a) Panoramic radiography shows a radiolucent lesion in the condylar process of the left mandible (black arrow). (b) Axial cone beam computed tomography confirms a radiolucent lesion with cortical breakthrough in the left mandible neck (white arrow). (c) T1-weighted magnetic resonance image after intravenous gadolinium contrast administration shows marked contrast enhancement of the bone marrow, loss of delineation of the cortex, and soft tissue extension in the adjacent pterygoidal (black asterisk) and masseter muscles (white asterisk). should therefore be preferred for the early diagnosis of acute osteomyelitis. ${ }^{16}$

\section{Adenomatoid Odontogenic Tumor}

An adenomatoid odontogenic lesion, formerly known as odontoameloblastoma, is predominantly of mixed density or radiopaque rather than radiolucent. Further discussion of this lesion is covered elsewhere in this issue. ${ }^{6}$

\section{Dentinogenic Ghost Cell Tumor}

A dentinogenic ghost cell tumor (DGCT), formerly known as cementifying fibroma, is considered a rare neoplastic variant of a calcifying odontogenic cyst. ${ }^{6,17}$ There are two distinct types of DGCT. The intraosseous DGCT tends to be locally aggressive with a mean age of 40 years. It mainly occurs in the canine to first molar region and usually presents as a painless bony swelling, although slight numbness and pain may occur. In contrast, the extraosseous form exhibits limited growth potential and usually occurs in the sixth decade. ${ }^{18}$ The anterior regions of the jaw bones are usually affected, typically at edentulous areas. It presents as a firm, painless nodule on the gingival or alveolar mucosa. ${ }^{19}$

Radiographically and on $\mathrm{CBCT}$, a variable appearance may be seen. Although the lesion is most often of mixed density with a variable degree of calcification, it could present as a completely radiolucent lesion. Most cases are unilocular, but multilocular lesions may be observed. These tumors are typically well defined, often expansile, and may result in resorption and divergence of roots of adjacent teeth. ${ }^{19}$

\section{Lesions with Preferential Pericoronal Location}

\section{Dentigerous Cyst}

A dentigerous cyst (DC), also known as a follicular cyst, is the second most common odontogenic cyst after a radicular cyst. It develops around a fully formed crown of an unerupted tooth. DC results from fluid accumulation between the follicular epithelium of the dental follicle and the crown of a developing tooth. ${ }^{4}$ When the lesion enlarges, the wall of the cyst therefore characteristically converges on the cementoenamel junction of the tooth (-Fig. S6). Microscopically we recognize nonspecific nonkeratinized, stratified squamous epithelium in the cyst lining. ${ }^{4}$

It is most commonly located at the third mandibular and maxillary molars or maxillary cuspids. ${ }^{4}$ It is often seen in the age group between 30 and 60 years, with a slight male predominance. ${ }^{4,16}$ Dentigerous cysts have a variable size, ranging from $<2 \mathrm{~cm}$ in diameter to cysts that cause marked expansion of the jaw. ${ }^{5}$ An enlarged asymmetric pericoronal space $>2.5 \mathrm{~mm}$ is considered suspicious for DC. ${ }^{4}$

Radiographically and on CBCT, DC typically consists of a well-defined unilocular radiolucency around the crown of an unerupted tooth surrounded by a rim of sclerosis. Large lesions may have undulating margins and may displace an involved unerupted tooth in the apical direction. The adjacent teeth may also be displaced, and resorption of their roots is more common than with other cysts. DC may 


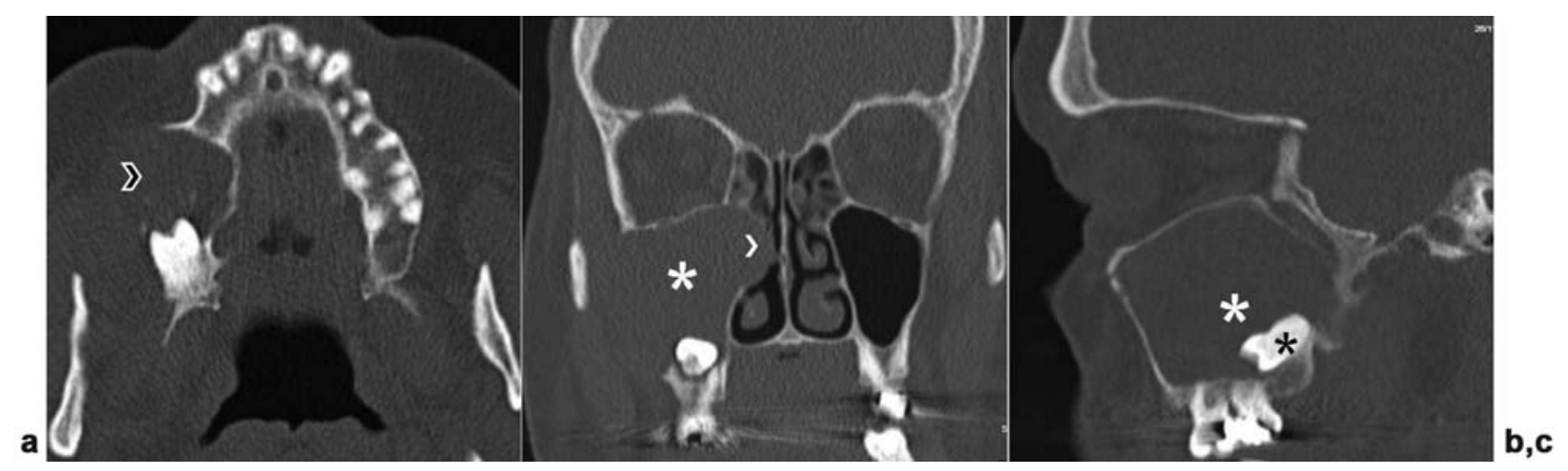

Fig. 4 Cone beam computed tomography of a dentigerous cyst. (a) Axial image. (b) Coronal image. (c) Sagittal image. Note an expansile radiolucent lesion (white asterisk) with cortical breakthrough at the posterolateral (black arrow) and medial wall of the maxillary sinus (white arrow), with obliteration of the meatus medius of the nasal cavity. There is also partial destruction of the right concha nasalis media. Note the intimate relationship with the crown of ectopic tooth 18 (black asterisk).

protrude into the maxillary antrum (-Fig. 4) or infratemporal fossa.

CBCT offers a better three-dimensional relationship between the cyst and the associated tooth. ${ }^{20,21}$ It also allows a more accurate evaluation of sclerosis of adjacent bone, cortical expansion, and breakthrough potential internal calcifications, as well the proximity to other important anatomical structures. ${ }^{22}$ This imaging guides further surgical planning.

On MRI, DC typically is homogeneous isointense with muscle on T1-weighted images and homogeneous or heterogeneous hyperintense on T2-weighted images. Rim enhancement may be seen. ${ }^{23}$ There is no periosteal reaction or associated soft tissue component. Ameloblastoma can occasionally develop within the walls of a DC. ${ }^{4}$

\section{Lesions with a Specific Location}

\section{Globulomaxillary Cyst}

In the past, the term globulomaxillary cyst was used to describe a so-called classic fissural cyst thought to occur secondary to epithelial entrapment in the globulomaxillary suture. ${ }^{24}$ Currently, it is believed a globulomaxillary cyst is a nonodontogenic cyst arising at the junction between the maxilla and premaxilla. ${ }^{25}$ The three main subtypes are nonalveolar, nasopalatine, and median palatal cysts. ${ }^{5}$ On imaging it has a typical homogeneous radiolucent appearance with the shape of an inversed pear or droplet. ${ }^{26}$

\section{Nasopalatine Duct Cyst}

A nasopalatine duct cyst (NPDC) is also known as an incisive canal cyst, cyst of palatine papilla, nasopalatine canal cyst, or median palatine cyst. It should be $>0.6 \mathrm{~cm}$ to distinguish it from the incisive foramen that is a normal anatomical feature. ${ }^{27}$

NPDC is a nonodontogenic cyst along fusion lines of the maxillary process. It arises from remnants of the embryonic nasopalatine duct that undergo spontaneous cystic degeneration and subsequently lead to cyst formation..$^{25}$

Histologically, most NPDCs contain nonkeratinized stratified squamous epithelium alone or in combination with other epithelia. Approximately $30 \%$ contain respiratory epithelium. ${ }^{28}$ It is most frequent in the fourth to sixth decades and more common in men. ${ }^{29}$

NPDC is found in the midline of the maxilla, between the roots of the central maxillary incisors that can be displaced or rarely slightly resorbed. ${ }^{4}$ It is mostly asymptomatic. A large lesion may present as swelling or discharge mucoid material. Patients may report a salty taste.

On plain radiography and CBCT, NPDC consists of a welldefined radiolucent lesion with sclerotic borders that may be round or oval. On panoramic views, the shadow of the nasal spine is sometimes superimposed on the cyst, giving it a heart shape. ${ }^{27}$ Midline location is the most useful clue to the correct diagnosis (-Fig. 5).

MRI usually shows homogeneous hyperintensity on both T1- and T2-weighted sequences. The T1 hyperintensity can be explained by the keratin or viscous fluid. ${ }^{23}$

\section{Nasolabial Cyst}

A nasolabial cyst is a developmental nonodontogenic type of soft tissue cyst occurring in the nasal alar region of the midface. This is in contrast to the NPDC, which is intraosseous located, in the anterior midpalatine region. ${ }^{30}$

Histopathologically, the cyst is lined with pseudostratified columnar epithelium and occasionally a stratified squamous epithelium. It is found most often in female adults in the fourth to fifth decade of life. Clinically, the lesion presents as a painless asymptomatic swelling in the nasolabial region. It results in obliteration of nasolabial sulcus, nasal vestibule, and maxillary labial sulcus. On palpation the lesion is soft and fluctuant in consistency. Patients generally report to a clinician for cosmetic reasons or a problem of nasal blockage. Diagnosis is established by clinical findings. Small nasolabial cysts are not depicted on plain radiographs.

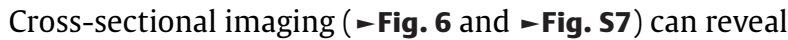
the extent and relation of the lesion to surrounding structures. ${ }^{30}$ Typically it is a well-defined round cystic mass in the nasal alar region, hypointense on T1-weighted images and hyperintense on T2-weighted images. ${ }^{31}$ 


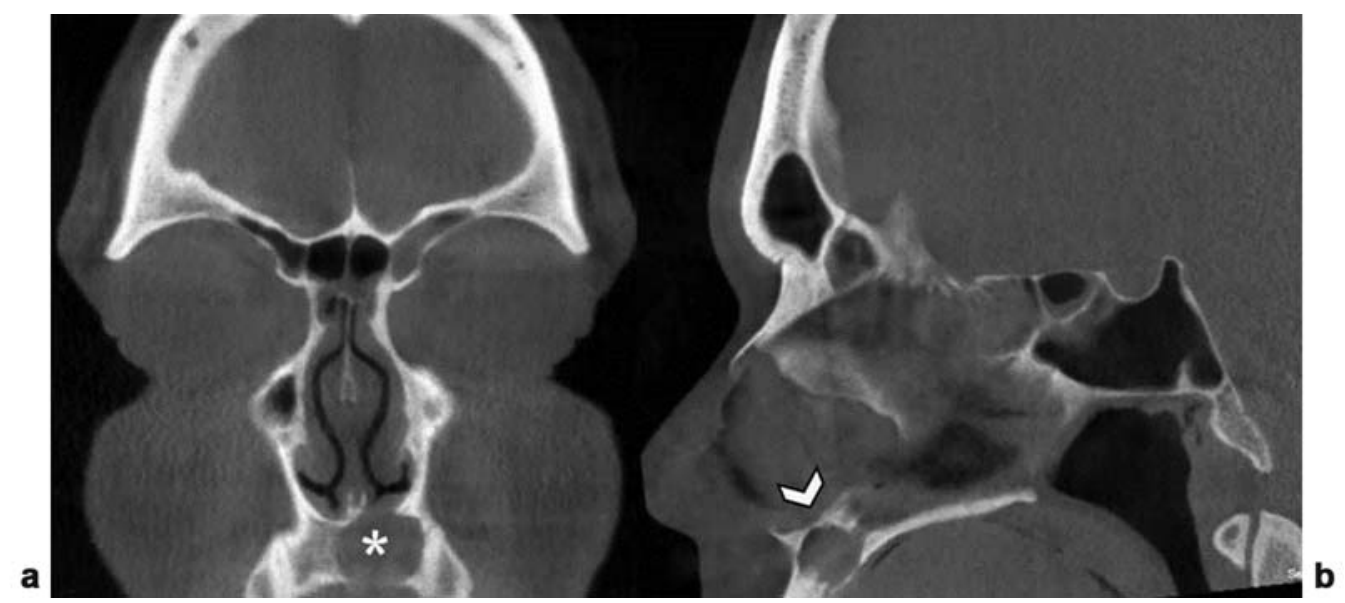

Fig. 5 Cone beam computed tomography of a nasopalatine duct cyst. (a) Coronal and (b) sagittal reconstructions show a well-defined radiolucent lesion (asterisk) in the midline of the palatum (white arrow). Note focal cortical thinning and even cortical breakthrough at the inferior border.

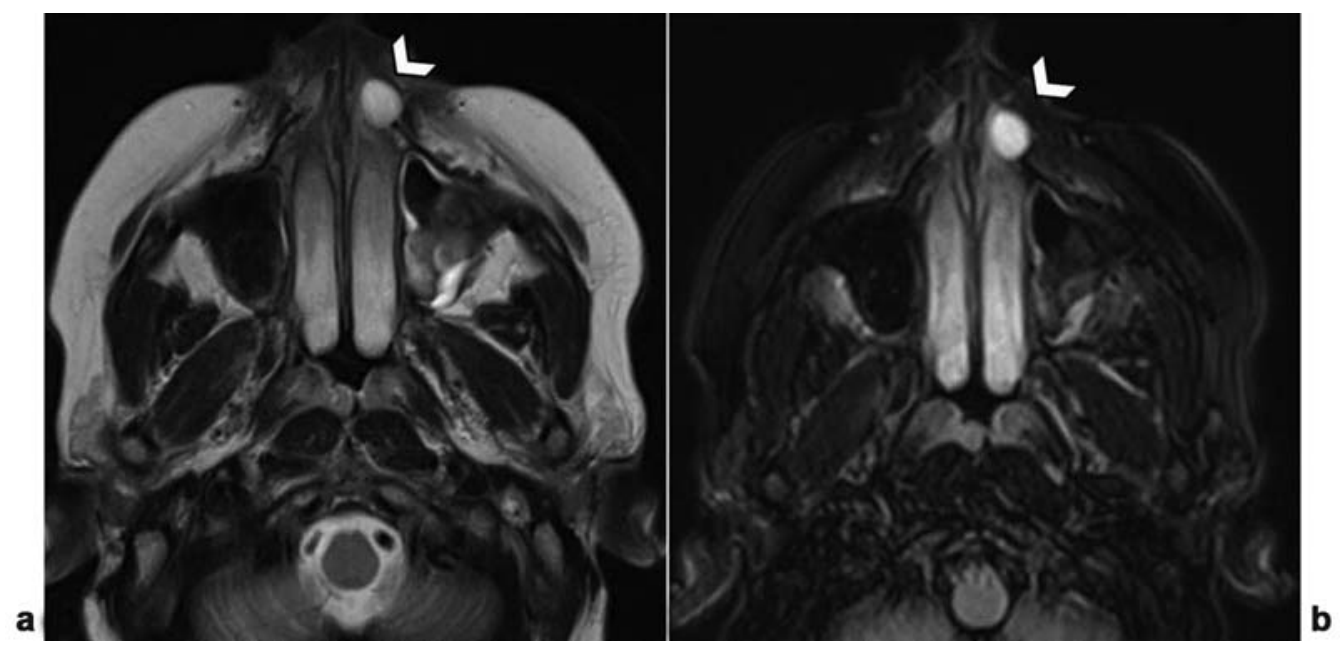

Fig. 6 Magnetic resonance imaging of a nasolabial cyst. (a) Axial T2-weighted image shows a well-delineated hyperintense lesion in the left anterior maxillary jaw in a 37-year-old man (white arrow). (b) T2-weighted image with fat saturation shows a lesion with a high signal in keeping with fluid contents (white arrow).

\section{Static Bone Cavity}

A static bone cyst is also known as a static bone cavity of the mandible, a Stafne bone cavity, or salivary gland inclusion defect. It is typically $<2 \mathrm{~cm}$ and usually an incidental finding on panoramic radiographs showing a well-delineated radiolucent lesion at the mandibular angle underneath the inferior alveolar canal. The cortical demarcation is usually thicker than those seen in other jaw cysts.

The lesion is secondary to pressure erosion of the adjacent submandibular gland and adjacent soft tissues. It is filled by a part of the submandibular gland, adjacent fat, and sometimes by muscle, lymphatic tissue, fat, and/or blood vessels. ${ }^{32}$ Strictly speaking, it is not a cyst because it does not contain fluid. Therefore, the term Stafne bone cavity is preferred. The lesion is more common in males than in females.

It is important to differentiate a Stafne bone cavity from other lesions within the mandible. The typical location at the mandibular angle at the location of the submandibular gland is the main clue to the correct diagnosis. However, rarely the lesion is located anteriorly (at the location of the sublingual salivary gland). In this scenario, it may be misdiagnosed and confused with other lesions. ${ }^{33}$

Panoramic radiography is sufficient for diagnosis, but occasionally CT or MRI can be performed in cases of atypical presentation to exclude an ameloblastoma or a traumatic bone cyst. ${ }^{32}$ Sialography, CT, CT sialography, and MRI have all been proposed as useful additional studies by showing the submandibular gland within the bone cavity. ${ }^{33}$

\section{Lesions That May Not Have Contact with Tooth}

\section{Residual Cyst}

A residual cyst is a persistent radicular cyst after extraction of the involved tooth. Except for the absence of a relationship with the tooth apex, the lesion has similar imaging features as a radicular cyst (-Fig. $\mathbf{5 8}$ ). 


\section{Ameloblastoma}

Ameloblastoma (AB) was formerly designated with the misnomer adamantinoma and adamantoblastoma. However, $A B$ is histologically unrelated to the adamantinoma of the bone. According to the latest revision of the World Health Organization 2017 classification, the formerly named calcifying odontogenic cyst also belongs to the group of $\mathrm{AB} .{ }^{6}$ A calcifying epithelial odontogenic cyst (also known as Pindborg's tumor) also belongs to the group of $\mathrm{AB}^{6}$

$A B$ is a neoplasm originating from the epithelium of the dental lamina. It arises from the reduced enamel epithelium after crown formation and therefore may mimic a dentigerous cyst. It is usually benign but locally aggressive. Malignant behavior is characterized by rapid growth, bone destruction, or very rarely distant metastases. $A B$ accounts for 10 to $18 \%$ of all odontogenic tumors and $1 \%$ of all lesions of the jaw. $A B$ occurs mostly in the third to fourth decade and has no sex predominance. ${ }^{4}$ Clinically, it is a painless slow-growing tumor and usually presents with jaw swelling. $\mathrm{AB}$ is mostly located in the molar-ramus region of the mandible. It can also be found in the molar-bearing portion of the maxilla and often involves the maxillary sinus. Lesions can sometimes be located in the pericoronal region with an unerupted tooth.

On panoramic view and CBCT, AB usually has well-defined margins, with no associated periosteal reaction. The lesion is typically radiolucent and can be multilocular or, less typically, unilocular. ${ }^{4}$ Loss of the lamina dura, erosion of the tooth apex, and displacement of the teeth are commonly seen. ${ }^{5}$ Large tumors may break through the cortex, with subsequent tumor extension into the adjacent soft tissues, best evaluated on cross-sectional imaging (-Fig. 7 and - Fig. S9). ${ }^{34}$

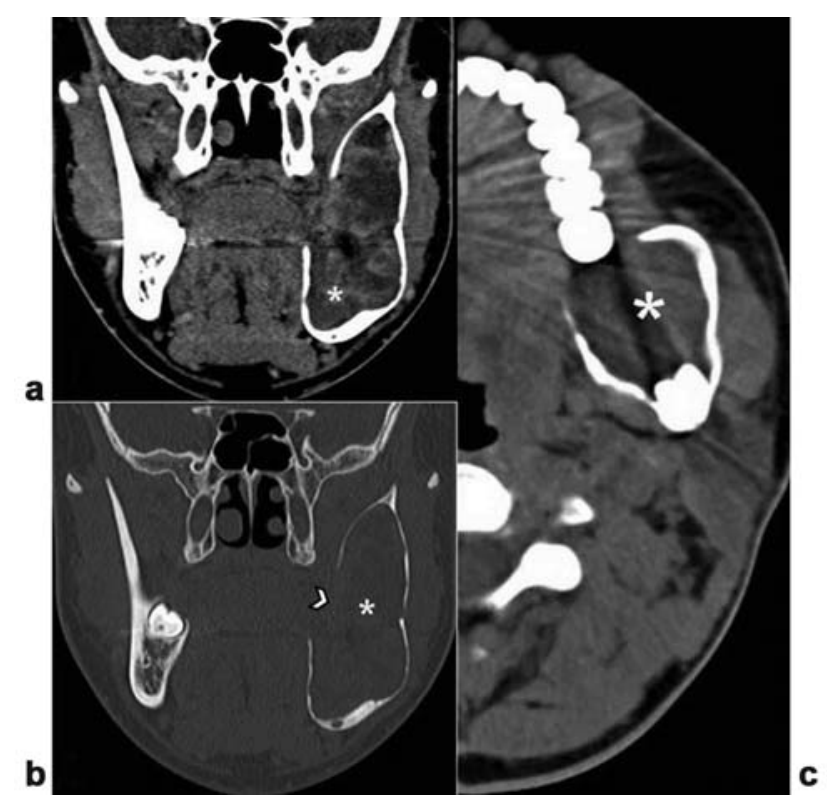

Fig. 7 Ameloblastoma. (a) Coronal computed tomography (CT) reconstruction in soft tissue window shows intralesional papillary projections (asterisk). (b) Coronal CT reconstruction in bone window. The mass (asterisk) is expansile, with associated bone remodeling, cortical thinning, and breakthrough (white arrow). (c) Axial CT in soft tissue window shows an expansile soft tissue mass (asterisk) in the left ramus ascendens of the mandible, with predominantly buccolingual extension.
On CT, AB appears as a hypodense lesion, with solid components that may enhance. Calcifications are rare. ${ }^{35}$

The lesion may contain septations, solid components, or papillary projections together with cystic spaces that reflect their gross pathology. ${ }^{4}$ A multiloculated mixed solid and cystic appearance is typical. Lesions are therefore heterogeneous on MR imaging, with irregularly thickened enhancing walls, often with solid papillary structures projecting into the lesion, with vivid enhancement. The solid components are intermediate intense on T1-weighted imaging and T2-weighted imaging; the cystic components are homogeneous hypointense on T1weighted imaging and hyperintense on T2-weighted imaging. ${ }^{36}$

\section{Giant Cell Lesion of Hyperparathyroidism}

A giant cell lesion of hyperparathyroidism, also known as a brown tumor, is associated with an underlying endocrinologic disturbance, characterized by increased serum parathyroid hormone (PTH). This latter leads to bone remodeling by means of osteoclastic resorption of bone. Histologically, the lesion contains a mixture of osteoclasts with fibrous tissue, old hemorrhage, and hemosiderin deposits. ${ }^{37}$

The mandible is the most frequent affected site in the maxillofacial area (-Fig. S10). Frequent clinical features include pain, swelling, facial asymmetry, and paresthesia. Multifocal involvement is seen in hyperparathyroidism, cherubism, or Noonan's syndrome. ${ }^{38}$

The most common radiographic findings are complete or partial loss of lamina dura of the teeth and ground-glass appearance of the jaw bones. Rarely, lytic lesions in the jaws may present as reparative giant cell granulomas, ${ }^{39}$ characterized by bone expansion and cortical thinning. ${ }^{15}$ The way to differentiate a brown tumor from a giant cell granuloma is to look at the serum PTH and calcium levels that are elevated in cases of hyperparathyroidism, and the age, whereas a giant cell granuloma preferentially occurs in young girls and women. ${ }^{16}$

\section{Osteomyelitis}

Osteomyelitis does not necessarily contact the teeth. Further discussion is found elsewhere in this issue.

\section{Direct Neoplastic Extension of Adjacent Head and Neck Tumors}

Malignant tumors arising from adjacent structures such as the gingiva or oral cavity may directly involve the jaw bones. Squamous cell carcinoma (SCC; -Figs. S11 and S12) is the most common malignancy involving the mandible or maxilla. SCC is the most common tumor of the oral cavity and preferentially affects men $>50$ years of age. SCC most often occurs in the mucosa overlying the posterior mandible, and it is related to consumption of alcohol and tobacco.

CT shows an aggressive soft tissue lesion with invasion of the floor of the mouth, alveolar ridge, or retromolar trigone. In advanced stages, secondary bone invasion can occur leading to the appearance of an ill-defined radiolucent lesion of the mandible on conventional radiographs; in advanced lesions, the "floating teeth" sign may be equally observed due to extensive mandibular infiltration. Direct tumor invasion may also present a pathologic fracture. ${ }^{16}$ 
On MRI, SCC displays a low to intermediate signal on T1weighted imaging, a moderately high signal on T2-weighted imaging and short tau inversion recovery sequences, and a moderate enhancement after injection of contrast media. DWI shows low ADC values. ${ }^{16}$

\section{Hematologic Disorders Involving the Jaws}

Although primary malignant bone tumors are not common in the mandible or maxilla, the mandible is susceptible to hematologic disorders such as multiple myeloma (MM), plasmacytoma, and lymphoma, along with metastatic tumors, because it contains abundant bone marrow space. ${ }^{3}$ $\mathrm{MM}$ is a malignancy originating from plasma cells. It is characterized by multiple or diffuse bone involvement. Less commonly single lytic lesions may occur. MM occurs most frequently in patients aged 40 to 70 years, with men more affected than women. ${ }^{40}$ The mandible is more affected than the maxilla. ${ }^{40}$ On imaging there are typically punched-out radiolucent lesions without circumferential bone reaction. The cortex of the mandible may be perforated but usually without associated bone expansion (-Fig. S13). Plasmacytoma is a localized form of MM and may affect the mandible.

Lymphoma may arise in the mandible and maxilla or be part of systemic lymphomatous disease. Non-Hodgkin's lymphoma is the most common type, of which the B-cell subgroup predominates. ${ }^{40}$ Primary Hodgkin's lymphoma is rare in the mandible, but the systemic form of Hodgkin's disease is not uncommon (8-23\%). ${ }^{40}$ There are no specific findings for lymphoma on imaging. A lytic destructive lesion can vary in size and be associated with loss of the lamina dura.

\section{Odontogenic Myxoma}

Odontogenic myxoma is a rare benign odontogenic tumor. It arises from mesenchymal cells and accounts for $17 \%$ of all odontogenic tumors. ${ }^{3}$ This lesion usually affects adults in the second and third decade of life. The mandibular premo- lar/molar region is a common location. The maxilla may be involved as well.

Radiographically, odontogenic myxoma usually presents as a well-defined radiolucent lesion with a distinct margin. ${ }^{15}$ Although benign, it may be locally aggressive with destruction of the cortex and resorption of the root of the adjacent tooth. This is more frequent when located in the maxilla.

Odontogenic myxomas vary in size. Smaller lesions may be unilocular; larger lesions tend to be multiloculated. The latter exhibits internal trabeculae with a "honeycomb" or "tennis-racket" internal architecture and may be associated with irregular calcifications. ${ }^{15}$

\section{Aneurysmal Bone Cavity}

Aneurysmal bone cyst $(\mathrm{ABC})$ is a benign intraosseous lesion composed of blood-filled cavities without an endothelial lining. It may develop after trauma or secondarily within a preexisting bone lesion (such as giant cell tumor, chondroblastoma, or fibrous dysplasia). There are also associations with venous obstruction. ${ }^{4} \mathrm{ABC}$ is found in children, with a female predominance. It most frequently affects the long tubular bones and spine (together 70\%) and usually presents as a painless enlarging swelling. It rarely involves the maxillofacial region. It accounts for just $1.5 \%$ of the nonodontogenic, nonepithelial cysts of the mandible, which is more frequently affected than the maxilla. ${ }^{41}$ On panoramic views, it may present as a well-defined expansile, unilocular, or multilocular radiolucency. Although it may appear entirely radiolucent on plain radiographs, CT or MRI may reveal internal ill-defined septations between cystic spaces with characteristic intralesional fluid-fluid levels. ${ }^{4} \mathrm{ABC}$ h have a propensity for marked osseous expansion, similar to the unilocular subtype of ameloblastoma.

\section{Calcifying Odontogenic Cyst}

The calcifying odontogenic cyst is also known as keratinizing and calcifying ameloblastoma, keratinizing and calcifying

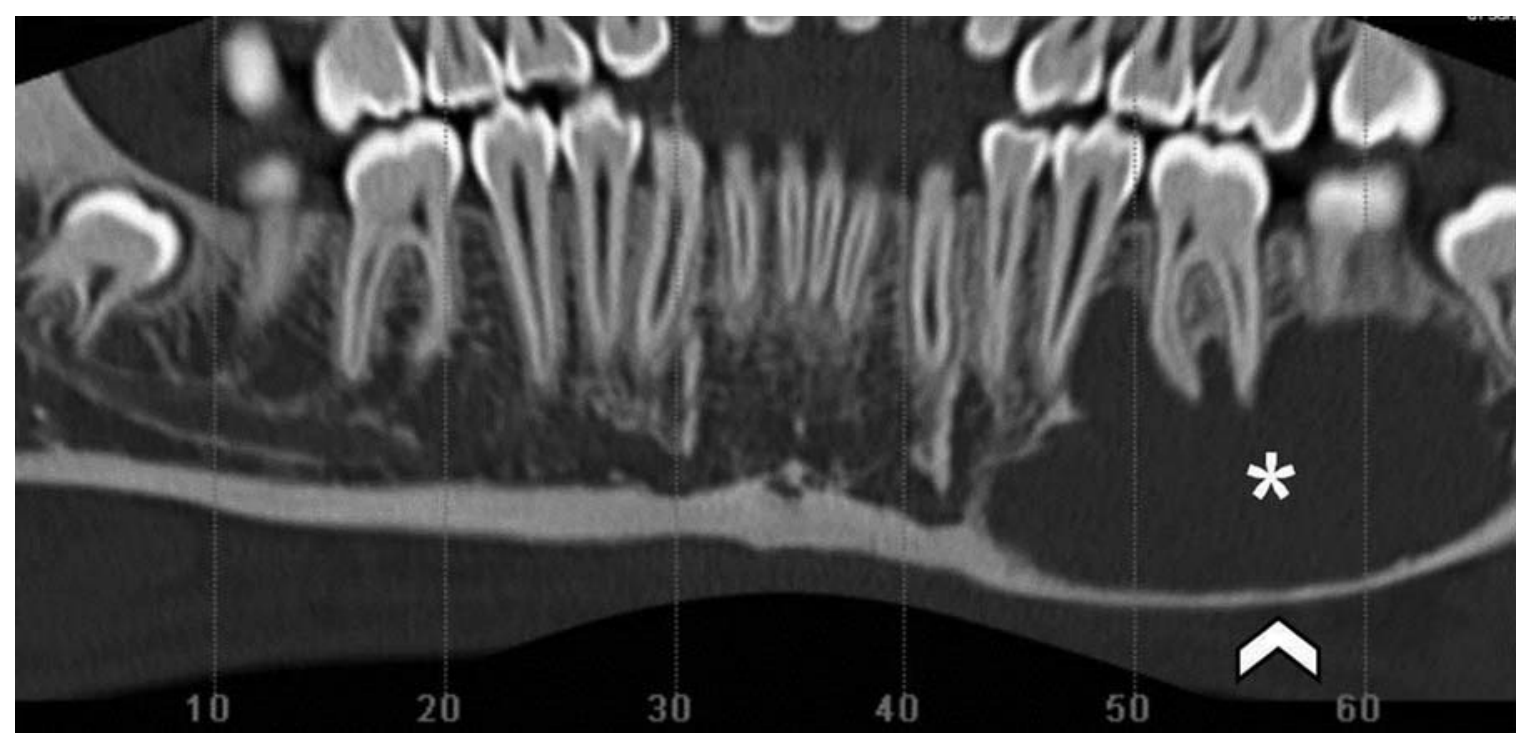

Fig. 8 Idiopathic bone cyst. Panoramic reconstruction of dental computed tomography shows a well-defined lesion in the left mandible (asterisk) with associated cortical thinning (white arrow). The lesion extends between the roots of the teeth. 
odontogenic cyst, or Gorlin's cysts. ${ }^{42}$ In a study by Irani et al, comprising 52 cases, radiographically 30 cases (58\%) showed a unilocular radiolucent area, and 22 cases (42\%) showed a mixed radiolucent/radiopaque appearance. ${ }^{43}$ Lesions of mixed density or radiopaque lesions are discussed elsewhere in this issue.

\section{Idiopathic Bone Cavity}

An idiopathic bone cavity (IBC) ( - Fig. 8 and - Fig. S14) is an empty bone cavity of unknown etiology. ${ }^{44}$ It was erroneously called a "traumatic bone cyst" for many years. However, it lacks the epithelial lining required of a cyst and is not always related to trauma. ${ }^{45}$ In case of trauma, there is a fracture or subluxation of teeth, as well as fracture of the mandible or maxilla that can cause a gap between the affected tooth and bone. A progressive radiolucent lesion around a tooth root with a history of a recent trauma supports the diagnosis of an IBC. ${ }^{3}$ IBC usually presents in the second decade of life and is typically located in the corpus of the mandible. ${ }^{45}$

Most often, IBC in the jaws presents as a unilocular radiolucent lesion that frequently scallops adjacent teeth roots that are vital without evidence of resorption or displacement. This important finding distinguishes IBC from more worrisome odontogenic lesions. ${ }^{46}$ The cyst may be slightly irregular in shape and have poorly defined borders, often referred to as an "preliminary pencil-sketch appearance." ${ }^{5}$ Because the cyst can contain serosanguinous fluid, CT and MRI may be helpful to evaluate the contents of the cyst. A relatively high density on CT correlates with blood products, but the density can be variable. On MRI, the bone cavity is filled with soft tissue that is continuous and on all sequences identical in signal with that of the mylohyoid muscle. ${ }^{47}$

\section{Conclusion}

Although imaging is not specific in characterization, the radiologist may be helpful in determining the density, location, and morphology of the lesion. The main imaging modalities are panoramic radiography and CBCT, and in selected cases MRI may be helpful for further characterization and additional information regarding soft tissue extension.

\section{Acknowledgment}

We would like to acknowledge and thank Bosmans Frederik, MD, for creating the illustrations in -Figs. $\mathbf{5 1}$ and $\mathbf{S 6 .}$

\section{References}

1 Sumi M, Ichikawa Y, Katayama I, Tashiro S, Nakamura T. Diffusion-weighted MR imaging of ameloblastomas and keratocystic odontogenic tumors: differentiation by apparent diffusion coefficients of cystic lesions. AJNR Am J Neuroradiol 2008;29(10): 1897-1901

2 Dunfee BL, Sakai O, Pistey R, Gohel A. Radiologic and pathologic characteristics of benign and malignant lesions of the mandible. Radiographics 2006;26(06):1751-1768

3 Chapman MN, Nadgir RN, Akman AS, et al. Periapical lucency around the tooth: radiologic evaluation and differential diagnosis. Radiographics 2013;33(01):E15-E32
4 Bharatha A, Pharoah MJ, Lee L, Tay KY, Keller A, Yu E. Pictorial essay: cysts and cyst-like lesions of the jaws. Can Assoc Radiol J 2010;61(03):133-143

5 Bernaerts A, Vanhoenacker FM, Hintjens J, et al. Tumors and tumor-like lesions of the jaw: radiolucent lesions. JBR-BTR 2006;89(02):81-90

6 Wright JM, Soluk Tekkesin M. Odontogenic tumors: where are we in 2017? J Istanb Univ Fac Dent 2017;51(03, Suppl 1):S10-S30

7 Snoeckx A, Vanhoenacker FM, Verhaert K, Chappelle K, Parizel PM. Gorlin-Goltz syndrome in a child: case report and clinical review. JBR-BTR 2008;91(06):235-239

8 Marcelis S, Van Damme S, Vanhoenacker FM. Odontogenic keratocyst of the mandible. 2018. Available at: https://www.eurorad. org/case/16132. Accessed February 25, 2020

9 Ali M, Baughman RA. Maxillary odontogenic keratocyst: a common and serious clinical misdiagnosis. J Am Dent Assoc 2003;134 (07):877-883

10 MacDonald-Jankowski DS. Keratocystic odontogenic tumour: systematic review. Dentomaxillofac Radiol 2011;40(01):1-23

11 Mosier KM. Lesions of the jaw. Semin Ultrasound CT MR 2015;36 (05):444-450

12 Borghesi A, Nardi C, Giannitto C, et al. Odontogenic keratocyst: imaging features of a benign lesion with an aggressive behaviour. Insights Imaging 2018;9(05):883-897

13 Sakamoto J, Kuribayashi A, Kotaki S, Fujikura M, Nakamura S, Kurabayashi T. Application of diffusion kurtosis imaging to odontogenic lesions: analysis of the cystic component. J Magn Reson Imaging 2016;44(06):1565-1571

14 de Carvalho LF, Lima CF, Cabral LA, Brandão AA, Almeida JD. Lateral periodontal cyst: a case report and literature review. J Oral Maxillofac Res 2011;1(04):e5

15 Kalkur C, Halim N, Sattur A, Burde K, Naikmasur V. Radiographical approach to multilocular radiolucent lesions of the jaws-a review. EC Dent Sci 2019;3:410-420

16 Avril L, Lombardi T, Ailianou A, et al. Radiolucent lesions of the mandible: a pattern-based approach to diagnosis. Insights Imaging 2014;5(01):85-101

17 Garcia Garcia B, Ruiz Masera JJ, Zafra Camacho FM, Centella Gutierrez $C$. Intraosseous dentinogenic ghost cell tumor: case report and treatment review. Rev Esp Cir Oral Maxilofac 2015;37(04):243-246

18 Li BBGY, Gao Y. Ghost cell odontogenic carcinoma transformed from a dentinogenic ghost cell tumor of maxilla after multiple recurrences. Oral Surg Oral Med Oral Pathol Oral Radiol Endod 2009;107(05):691-695

19 Agrawal Y, Naidu GS, Makkad RS, et al. Dentinogenic ghost cell tumor-a rare case report with review of literature. Quant Imaging Med Surg 2017;7(05):598-604

20 Deana NF, Alves N. Cone beam CT in diagnosis and surgical planning of dentigerous cyst. Case Rep Dent 2017;2017:7956041

21 Mahesh BS, P Shastry S, S Murthy P, Jyotsna TR. Role of cone beam computed tomography in evaluation of radicular cyst mimicking dentigerous cyst in a 7-year-old child: a case report and literature review. Int J Clin Pediatr Dent 2017;10(02):213-216

22 Ahmad M, Jenny J, Downie M. Application of cone beam computed tomography in oral and maxillofacial surgery. Aust Dent J 2012;57 (Suppl 1):82-94

23 Hisatomi M, Asaumi J, Konouchi H, Shigehara H, Yanagi Y, Kishi K. MR imaging of epithelial cysts of the oral and maxillofacial region. Eur J Radiol 2003;48(02):178-182

24 Reddy L, Jain V, Mittal S, Gupta S. Full moon shaped unusual appearance of a globulomaxillary cyst: a case report. J Indian Acad Oral Med Radiol 2017;29(01):50

25 De Cuyper K, Vanhoenacker FM, Hintjens J, Verstraete KLPP, Parizel PM. Nasopalatine duct cyst. JBR-BTR 2008;91(04):179

26 Audion M, Siberchicot F. Kyste globulomaxillaire. Rev Stomatol Chir Maxillofac 2010;111(01):25-26

27 Srivastava S, Misra N, Agarwal R, Pandey P. Nasopalatine canal cyst: often missed. BMJ Case Rep 2013;2013:2-4 
28 Swanson KS, Kaugars GE, Gunsolley JC. Nasopalatine duct cyst: an analysis of 334 cases. J Oral Maxillofac Surg 1991;49(03):268-271

29 Cecchetti F. Nasopalatine duct cyst: report of 52 cases in a retrospective epidemiological study. J Surg (Northborough) 2013;1(02):14

30 Narain S. Nasolabial cyst: clinical presentation and differential diagnosis. J Maxillofac Oral Surg 2015;14(Suppl 1):7-10

31 Ocak A, Duman SB, Bayrakdar IS, Cakur B. Nasolabial cyst: a case report with ultrasonography and magnetic resonance imaging findings. Case Rep Dent 2017;2017:4687409

32 More CB, Das S, Gupta S, Patel P, Saha N. Stafne's bone cavity: a diagnostic challenge. J Clin Diagn Res 2015;9(11):ZD16-ZD19

33 Taysi M, Ozden C, Cankaya B, Olgac V, Yıldırım S. Stafne bone defect in the anterior mandible. Dentomaxillofac Radiol 2014;43 (07):20140075

34 Vanhoenacker FM, Chapelle K, Dekeyzer S. Ameloblastoma. JBRBTR 2009;92(06):300

35 Lascane Nda S, Sedassari BT, Alves FdeA, Gallottini MH, de Sousa SC. Peripheral ameloblastoma with dystrophic calcification: an unusual feature in non-calcifying odontogenic tumors. Braz Dent J 2014;25(03):253-256

36 Asaumi J, Hisatomi M, Yanagi Y, et al. Assessment of ameloblastomas using MRI and dynamic contrast-enhanced MRI. Eur J Radiol 2005;56(01):25-30

37 Costa FR, Esteves C, Bacelar MT. Benign mandibular lesions : a pictorial review. Acta Radiol Port 2016;28(108):25-34
38 Eyselbergs M, Vanhoenacker F, Hintjens J, Dom M, Devriendt K, Van Dijck H. Unilateral giant cell lesion of the jaw in Noonan syndrome. JBR-BTR 2014;97(02):90-93

39 Rai S, Rattan V, Bhadada SK. Giant cell lesions associated with primary hyperparathyroidism. J Maxillofac Oral Surg 2015;14(04):930-934

40 Weber AL, Bui C, Kaneda T. Malignant tumors of the mandible and maxilla. Neuroimaging Clin N Am 2003;13(03):509-524

41 Devi P, Thimmarasa V, Mehrotra V, Agarwal M. Aneurysmal bone cyst of the mandible: a case report and review of literature. J Oral Maxillofac Pathol 2011;15(01):105-108

42 Uchiyama Y, Akiyama H, Murakami S, et al. Calcifying cystic odontogenic tumour: CT imaging. Br J Radiol 2012;85(1013):548-554

43 Irani S, Foroughi F. Histologic variants of calcifying odontogenic cyst: a study of 52 cases. J Contemp Dent Pract 2017;18(08):688-694

44 Bernaerts A, Vanhoenacker FM, Geenen L, Quisquater G, Parizel PM. Conventional dental radiology: what the general radiologist needs to know. JBR-BTR 2006;89(01):23-32

45 Dekeyzer S, Vanhoenacker FM, Chapelle K. Idiopathic bone cyst of the mandible. JBR-BTR 2010;93(03):132-133

46 Horne RP, Meara DJ, Granite EL. Idiopathic bone cavities of the mandible: an update on recurrence rates and case report. Oral Surg Oral Med Oral Pathol Oral Radiol 2014;117(02):e71-e73

47 Ertas E, Atici M, Kalabalik F, Ince O. Investigation and differential diagnosis of Stafne bone cavities with cone beam computed tomography and magnetic resonance imaging: report of two cases. J Oral Maxillofac Radiol 2015;3(03):92 